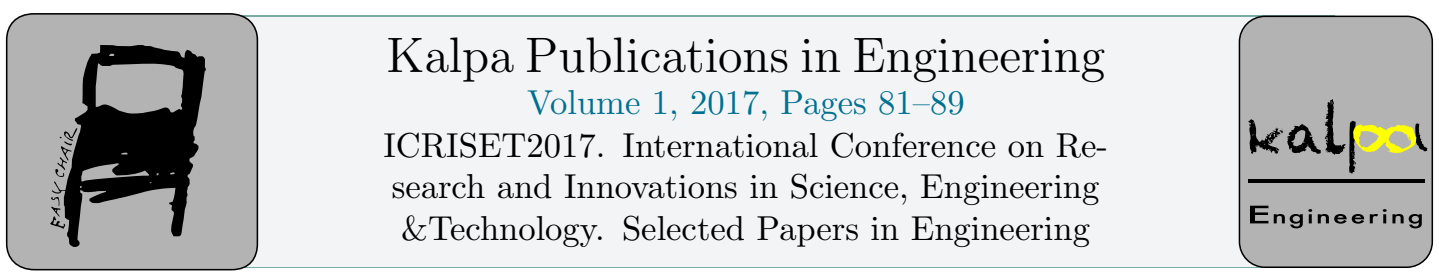

\title{
Optimal Coordination of Directional Overcurrent Relays Using AI Algorithms and Comparison.
}

\author{
Gaurav Darji, Ajay Patel, Rashesh Mehta \\ Department of Electrical Engineering, Birla Vishvakarma Mahavidyalaya \\ V.V.Nagar, Anand, India
}

\begin{abstract}
- for identification of fault in time, with effectiveness and also to isolate the faulted part from the system to keep away from probable outages in a power system, the precise coordination of Directional Overcurrent Relays (DOCRs) is required. The coordination of DOCRs is assessed as optimization problem with containing complex nonlinear constraints. In this paper, several nature inspired AI techniques are implemented for the optimum solution of DOCR coordination problem. Fine tuning of presented AI algorithm is done to get the optimum possible results. Also the obtained results using the proposed methods are hybridized with the nonlinear programming technique for obtaining global best solution. All four algorithms represented for a case study system are compared with each other on the basis of Fitness of solution, convergence time of an algorithm for solution and on the basis of complexities presented by them in the way of solution. The results obtained present that with fine tuning of separate algorithm and using hybridization approach leads to the optimum as well as feasible solution within the boundary limits.
\end{abstract}

Keywords- directional overcurrent relays,relay coordination, AI algorithms, GA, CSA, FFA, HSA,NLP,parameters tuning.

\section{Introduction}

Protective relays play an important role in the power system security. Optimum operation of relays is essential for the reliability and protect-ability in power system. The concept of coordination of relays consists of finding the setting of the relays for the operating parameter so that the faults can be well recognized and isolated as quickly as possible from the reliability point of view. Generally with radial connected distribution system Directional Overcurrent Relays (DOCRs) are more preferred as a primary protection and in Transmission system as a backup protection. The concept of optimum coordination of relays is directed to get the two optimum settings, Time Multiplier Setting (TMS) and Plug Setting (PS) with consideration of problem boundary limits and the coordination constraints criteria.

In this paper a different approach towards the optimum solution of relay coordination is assessed. As the most important protection is provided by primary protection only, the major concentration is made on the optimization of time of operation (TOP) of the primary protecting relay. In achieving so hybridization of different AI algorithm with NLP is made and also the consideration on the coordination time interval (CTI) between P/B pair is secured to lowest possible values. Various four algorithms e.g. Genetic Algorithm (GA), Cuckoo Search Algorithm (CSA), Firefly algorithm (FFA) and Harmony Search Algorithm (HSA) are analyzed and finely tuned to get the optimum possible TMS and PS by themselves only. Further to get the global best optimum each of the presented algorithms is

A. Shukla, J.M. Patel, P.D. Solanki, K.B. Judal, R.K. Shukla, R.A. Thakkar, N.P. Gajjar, N.J. Kothari, S. Saha, S.K. Joshi, S.R. Joshi, P. Darji, S. Dambhare, B.R. Parekh, P.M. George, A.M. Trivedi, T.D. Pawar, M.B. Shah, V.J. Patel, M.S. Holia, R.P. Mehta, J.M. Rathod, B.C. Goradiya and D.K. Patel (eds.),

ICRISET2017 (Kalpa Publications in Engineering, vol. 1), pp. 81-89 
hybridized with NLP. Succeeding a detailed comparison among all the algorithms is tabulated on the basis of Result optimization, Time of convergence, Parameter tuning requirements and consistency in the results. Also the statistical analysis for all the algorithms is presented in the form of graph for a set of 50 simulations each.

Detailed objective function and constraint set formulation is presented in Section 2 and Various AI algorithms are described in Section 3. A 9-bus case study as an implementation of proposed methodology is analyzed in Section 4. The obtained results are summarized and comparison among them is tabulated with conclusion in Section 5.

\section{Problem Formulation}

$T M S$ and Plug Setting $(P S)$ are the two parameters based on which relay coordination problem is formulated. The relay coordination problem includes Objective Function $(O F)$ and constraints. That must satisfy all essential rudiments.

\subsection{Objective Function formulation}

The DOCR coordination problem is formulated as an optimization problem. All the operating times of primary relays are summed up together to formulate $O F$, which is to be minimized.

$$
O F=\operatorname{Min} \sum_{j=1}^{n}\left(W^{j} T_{k}^{j}\right)
$$

Where, $T_{k}^{j}$ is operating time of relay $\mathrm{R}_{j}$ for zone $\mathrm{k}$ close in fault, $W^{j}$ is weight factor for the operating time of relay $\mathrm{R}_{j}, n$ is number of relays. $W^{j}$ is a weighting factor representing the likelihood of a fault occurring on $\mathrm{j}^{\text {th }}$ line in zone $\mathrm{k}$ of power network. Generally the value of $W^{j}$ is set 1 [3-7].

\subsection{Formulation of constraints}

The following five sets of constraints must be satisfied owing to optimize the $O F$.

\subsubsection{Bounds on TMS}

Whenever the fault level makes a value equal to or greater than the current setting of a relay, definite time delay is offered by TMS before the relay trips. The TMS bound of relay can be defined as:

$$
T M S_{j, \min } \leq T M S \leq T M S_{j, \max }
$$

The ranges of TMS value are incessantly from 0.025 to 1.2 [6].

\subsubsection{Bounds on PS}

The determination of $P S$ is based on two parameters, the maximum load current $\left(\mathrm{I}_{\operatorname{lmax}}\right)$ and the minimum fault current $\left(\mathrm{I}_{\text {fmin }}\right)$, for assurance that the relays will not mal-operate. Also, the relay must be sensitive to the smallest fault current [2], [6], [7].

The bound on $P S$ of relay can be stated as:

$$
P S_{j, \min } \leq P S \leq P S_{j, \max }
$$

Where $P S_{j, \min }$ and $P S_{j, \max }$ are minimum and maximum value of $P S$ of relay $\mathrm{R}_{j}$. 


\subsubsection{Coordination Time Interval (CTI)}

The required time period for sustaining selectivity between $\mathrm{P} / \mathrm{B}$ pair of relay is known as CTI. The CTI depends upon several factors like the relay types, relay inaccuracy, operating time of CB and safety margin [5], [6]. It can be stated as:

$$
T_{i, k}-T_{j, k} \geq C T I
$$

Where $T_{i, k}$ is the operating time of the backup relay $R_{i}$ and $T_{j, k}$ is for the primary relay $\mathrm{R}_{\mathrm{j}}$. the $C T I$ value varies from 0.2 to 0.5 seconds.

\subsubsection{Bounds on TOP of Relay}

The relay requires definite minimum amount of time to operate considering the transient maloperations as well as it should not to be allowable to take far-reaching time to operate. It can be stated as:

$$
T_{j, \min } \leq T \leq T_{j, \max }
$$

Where $T_{j, \min }$ and $T_{j, \max }$ are minimum and maximum operating time of relayR .

\subsubsection{Relay Characteristics:}

The relay characteristic adopted in this study, nonlinear and most preferable inverse definite minimum time (IDMT) characteristic function based on the IEC standard is expressed in (6).

$$
T O P_{j}=\frac{0.14 \times T M S_{j}}{\left({ }^{I_{R, j}} / P S_{j}\right)^{0.02}-1}
$$

Where, $T O P_{j}, P S_{j}$ and $T M S_{j}$ are operating time, plug setting and time multiplier setting of relayR $\mathrm{R}_{j}$ respectively. $I_{\text {relay }}$ is fault current seen by relayR $R_{j}$.

\section{AI Algorithms}

\subsection{Cuckoo Search Algorithm}

Different species of the cuckoo birds follows a distinct type of progeny parasitism. Unlike other living generations they don't build their own asylum to provide the fittest safety to next generations instead they make a search of various fittest nests of other species to lay their eggs [9]. This search of finding fittest nest for laying eggs is really magnificent as if the host birds come across the knowledge of an unfamiliar egg in their nest they either throw off alien eggs or either abandon their nest. Inspiring from this distinct formulation of the survival of the fittest, an optimization technique called cuckoo search algorithm has developed. As cuckoo species search for fittest nest out of several choices similar an optimization objective problem can be formulated to get global optimum solution within the problem boundaries and the multiple constraints set. The progeny progress showed by cuckoo species depends on some policies like a) unambiguous brood parasitism b) breed assistance c) captivating over host nests [8].

\subsection{Firefly Algorithm}

Based on the flashing patterns and the behavior of fireflies in nature, the Firefly Algorithm (FA) was first introduced by Xin-She Yang in 2007 and 2008 at Cambridge University. According to study, nearly two thousand different types of species of firefly are observed, and they can be distinguished from each other by their specific production of rhythmic and short flashes. Point to be noticed in this phenomenon is that for a specific species of firefly generation the pattern is observed with unique 
flashes generation. The attraction of both the males and females to each other depends on several factors like i) the rhythm in which the flashes produced ii) flashing rate iii) time recorded until completion of flash pattern observation. Observing different male flash patterns Female species respond individually for further production process [10]. This newly developed nature inspired algorithm can also be implemented for relay coordination problem.

\subsection{Harmony Search Algorithm}

Based on the musicians skill of selection of rhythmic tunes the harmony search as an inspiration in soft computing algorithm developed. The musicians improvise the best possible harmony tune in terms of aesthetics. The key point of notice in HS is, the HS algorithm does not require initial values and instead of performing a gradient search it uses a random search, so derivative information is unnecessary. Both the concepts of the harmony selection in the virtue of the music formulation and the optimization are analogous to each other. As harmony search is the meta-heuristic process of formulation of music by selection of perfect tunes and rejecting previously recorded unproductive tunes and repeating process until best tune is obtained. The concept of optimization repeats the same story of having an operating function which is to be tuned in the boundaries of the constraints set until the global best solution is achieved. [11].

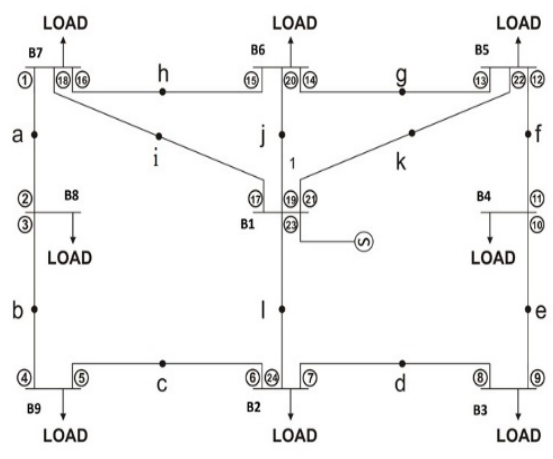

Figure 1: Single-line diagram of a nine-bus system

\section{Implementation of proposed method}

Various AI algorithms projected in literature are implemented on a case study of 9-bus system. Various parameters are tuned in the analysis to find the best optimum promising parameters for getting optimum TOP and CTI of DOCRs. All the relays considered as the directional IDMT relays in the case study. The TMS value lies in the interval of $(0.025,1.2)$ [6]. Both the CTI and minimum operating time of each relay are assumed to be 0.2 second.

\section{Case Study: 9- bus system}

In this case study a 9 bus (B1 to B9) system with a single bus supply is taken. Bus 1 has the only generation unit in the taken system. The rated supply is at 100 MVA and $33 \mathrm{KV}$ base values. For simplicity purpose the resistances of generating unit as well as all the tie lines are neglected. Only the reactance for both is considered as value of $\mathrm{j} 0.1$ p.u. for source and j0.2 p.u. for tie lines [6].

The information regarding the load currents at the buses, the P/B relay association for different fault points as taken in the study, the minimum and the maximum fault currents and also the maximum load currents through the relays are given in [6]. 


\section{i) Solution Initializing}

The objective function is formulated as from the given in the equation (1). In which first $\mathrm{x} 1$ to $\mathrm{x} 24$ are taken as the $T M S$ of the 24 IDMT relays respectively and $\mathrm{x} 25$ to $\mathrm{x} 48$ onwards are taken as the PS. The minimum TOP and the CTI are taken as 0.2 second.

Equation (2) and (6) are used to form coordination constraints. Equation (3) and (6) for relay TOP constraints and bounds $\left(\mathrm{TMS}_{\mathrm{j}}, \mathrm{PS}_{\mathrm{j}}\right.$ ) are formed by equation (4) and (5)

\section{ii)Finding Initial Solution:}

Following above stated methodology initial solutions of TMS and PS are simulated by the various AI algorithms proposed (GA, CSA, FFA and $H S A)$.

The obtained TMS and PS for the 24 IDMT relays simulated by the GA, CSA, FFA and HSA are given in the table no. 1, no. 2 , no. 3 and no. 4 respectively in $2^{\text {nd }}$ and $3^{\text {rd }}$ column.

\section{iii) Hybridization with NLP method:}

Obtaining the initial TMS and PS from the base algorithms these solutions are given as the initial startup point for the NLP solution having the same $O F$ and the constraints set. MATLAB NLP toolbox is used for solution. Results are shown with hybridization in the tables as explained above.

\begin{tabular}{|c|c|c|c|c|}
\hline \multirow{2}{*}{ Relay } & \multicolumn{2}{|c|}{ GA } & \multicolumn{2}{c|}{ GA-NLP } \\
\cline { 2 - 5 } & TMS & PS & TMS & PS \\
\hline $\mathbf{1}$ & 0.2239 & 0.3044 & 0.0557 & 1.4373 \\
$\mathbf{2}$ & 0.0383 & 0.8699 & 0.0398 & 0.8272 \\
$\mathbf{3}$ & 0.1119 & 0.728 & 0.0461 & 1.1472 \\
$\mathbf{4}$ & 0.4592 & 0.0054 & 0.0538 & 0.8232 \\
$\mathbf{5}$ & 0.1943 & 0.2114 & 0.0462 & 0.7239 \\
$\mathbf{6}$ & 0.1444 & 0.5136 & 0.0524 & 1.4466 \\
$\mathbf{7}$ & 0.1024 & 0.886 & 0.0604 & 1.104 \\
$\mathbf{8}$ & 0.1017 & 0.393 & 0.0445 & 0.767 \\
$\mathbf{9}$ & 0.5187 & 0.0054 & 0.0538 & 0.8227 \\
$\mathbf{1 0}$ & 0.4018 & 0.0128 & 0.0511 & 0.969 \\
$\mathbf{1 1}$ & 0.0856 & 0.5578 & 0.0465 & 0.6583 \\
$\mathbf{1 2}$ & 0.4588 & 0.7994 & 0.0488 & 1.0494 \\
$\mathbf{1 3}$ & 0.3296 & 0.0846 & 0.0532 & 1.1859 \\
$\mathbf{1 4}$ & 0.1969 & 0.3383 & 0.0521 & 1.3914 \\
$\mathbf{1 5}$ & 0.055 & 1.3505 & 0.0639 & 0.937 \\
$\mathbf{1 6}$ & 0.1132 & 0.3292 & 0.0689 & 0.6984 \\
$\mathbf{1 7}$ & 0.0856 & 1.1053 & 0.0767 & 1.112 \\
$\mathbf{1 8}$ & 0.041 & 1.1033 & 0.0409 & 1.1086 \\
$\mathbf{1 9}$ & 0.0866 & 1.0272 & 0.0782 & 1.0348 \\
$\mathbf{2 0}$ & 0.0474 & 1.0272 & 0.0474 & 1.0272 \\
$\mathbf{2 1}$ & 0.0859 & 1.1034 & 0.0768 & 1.1112 \\
$\mathbf{2 2}$ & 0.0411 & 1.1035 & 0.041 & 1.1035 \\
$\mathbf{2 3}$ & 0.074 & 1.2663 & 0.074 & 1.2663 \\
$\mathbf{2 4}$ & 0.0483 & 1.2364 & 0.0261 & 1.3457 \\
\hline $\mathbf{O F}$ & \multicolumn{2}{|c|}{8.93} & \multicolumn{2}{|c|}{4.8} \\
\hline
\end{tabular}

Table 1: TMS and PS of Relays GA and GA-NLP

\section{iv) Calculating time of operation of the relays:}

Obtaining the TMS and PS of all 24 relays as a point from $\mathrm{x} 1$ to $\mathrm{x} 48$, are applied to the $O F$. The result shows the total operating time and the separate primary operation time for all 24 relays are calculated with the use of TMS and PS obtained by various AI methods to get respective TOP of relays. The TOP for all relays obtained by GA, CSA, FFA, HSA and also the hybridized outcome with NLP are given in the table no. 5.

v) Summary:

As a summary of results the base AI algorithm results in local best solutions while when they are hybridized with NLP the solutions obtained are the optimum possible. 
There are two basic parameters offered by CSA i) Number of nests (n) and ii) Discovery rate of alien eggs (pa).

A fine tuning of both has been done to get the fittest parameter in obtaining our system problem.

\begin{tabular}{|c|c|c|c|c|}
\hline \multirow{2}{*}{ Relay } & \multicolumn{2}{|c|}{ CSA } & \multicolumn{2}{c|}{ CSA-NLP } \\
\cline { 2 - 5 } & TMS & PS & TMS & PS \\
\hline $\mathbf{1}$ & 0.0756 & 0.7388 & 0.0585 & 1.3073 \\
$\mathbf{2}$ & 0.0386 & 0.8608 & 0.0508 & 0.57 \\
$\mathbf{3}$ & 0.0537 & 0.8872 & 0.0383 & 1.499 \\
$\mathbf{4}$ & 0.0483 & 0.9883 & 0.0383 & 1.3921 \\
$\mathbf{5}$ & 0.0462 & 0.7248 & 0.0464 & 0.7193 \\
$\mathbf{6}$ & 0.0666 & 0.8972 & 0.0745 & 0.6883 \\
$\mathbf{7}$ & 0.0557 & 1.291 & 0.0732 & 0.7187 \\
$\mathbf{8}$ & 0.0522 & 0.5901 & 0.0386 & 0.9388 \\
$\mathbf{9}$ & 0.044 & 1.1469 & 0.053 & 0.8455 \\
$\mathbf{1 0}$ & 0.0524 & 0.9264 & 0.0538 & 0.8841 \\
$\mathbf{1 1}$ & 0.0525 & 0.5375 & 0.0383 & 0.8695 \\
$\mathbf{1 2}$ & 0.051 & 0.9721 & 0.058 & 0.7678 \\
$\mathbf{1 3}$ & 0.0724 & 0.6215 & 0.0695 & 0.6857 \\
$\mathbf{1 4}$ & 0.0644 & 0.9188 & 0.0526 & 1.3663 \\
$\mathbf{1 5}$ & 0.0624 & 0.9839 & 0.063 & 0.9642 \\
$\mathbf{1 6}$ & 0.055 & 1.1154 & 0.0756 & 0.5592 \\
$\mathbf{1 7}$ & 0.0761 & 1.135 & 0.077 & 1.1035 \\
$\mathbf{1 8}$ & 0.0379 & 1.2252 & 0.0309 & 1.5601 \\
$\mathbf{1 9}$ & 0.0764 & 1.0988 & 0.0773 & 1.0682 \\
$\mathbf{2 0}$ & 0.0312 & 1.7829 & 0.0348 & 1.5776 \\
$\mathbf{2 1}$ & 0.0769 & 1.1045 & 0.076 & 1.1382 \\
$\mathbf{2 2}$ & 0.0333 & 1.4359 & 0.041 & 1.1033 \\
$\mathbf{2 3}$ & 0.0733 & 1.298 & 0.074 & 1.2663 \\
$\mathbf{2 4}$ & 0.0253 & 1.3843 & 0.0252 & 1.3902 \\
\hline OF & \multicolumn{2}{|c|}{4.8008} & \multicolumn{2}{|c|}{4.8} \\
\hline $\mathbf{T}$ & \multicolumn{2}{|c|}{$\mathbf{2}$} & & \\
\hline
\end{tabular}

Table 2: TMS and PS of Relays CSA and CSA-NLP

The FFA has some more parameters to be tuned in like i) Population size (n), ii) no. of iterations (N), iii) Randomness ( $\alpha$ ), iv) Brightness coefficient $(\beta)$, v) Absorption coefficient $(\gamma)$.

\begin{tabular}{|c|c|c|c|c|}
\hline \multirow{2}{*}{ Relay } & \multicolumn{2}{|c|}{ FFA } & \multicolumn{2}{c|}{ FFA-NLP } \\
\cline { 2 - 5 } & TMS & PS & TMS & PS \\
\hline $\mathbf{1}$ & 0.168 & 0.53 & 0.076 & 0.738 \\
$\mathbf{2}$ & 0.054 & 0.531 & 0.054 & 0.514 \\
$\mathbf{3}$ & 0.332 & 0.068 & 0.054 & 0.884 \\
$\mathbf{4}$ & 0.098 & 0.403 & 0.054 & 0.823 \\
$\mathbf{5}$ & 0.16 & 0.237 & 0.054 & 0.562 \\
$\mathbf{6}$ & 0.204 & 0.352 & 0.076 & 0.664 \\
$\mathbf{7}$ & 0.176 & 0.465 & 0.076 & 0.664 \\
$\mathbf{8}$ & 0.08 & 0.59 & 0.054 & 0.56 \\
$\mathbf{9}$ & 0.264 & 0.108 & 0.054 & 0.822 \\
$\mathbf{1 0}$ & 0.293 & 0.184 & 0.054 & 0.884 \\
$\mathbf{1 1}$ & 0.065 & 0.555 & 0.054 & 0.514 \\
$\mathbf{1 2}$ & 0.244 & 0.812 & 0.058 & 0.768 \\
$\mathbf{1 3}$ & 0.128 & 0.455 & 0.074 & 0.594 \\
$\mathbf{1 4}$ & 0.229 & 0.33 & 0.069 & 0.778 \\
$\mathbf{1 5}$ & 0.17 & 0.329 & 0.076 & 0.633 \\
$\mathbf{1 6}$ & 0.086 & 0.482 & 0.061 & 0.918 \\
$\mathbf{1 7}$ & 0.074 & 1.236 & 0.074 & 1.203 \\
$\mathbf{1 8}$ & 0.038 & 1.216 & 0.033 & 1.429 \\
$\mathbf{1 9}$ & 0.079 & 1.065 & 0.076 & 1.13 \\
$\mathbf{2 0}$ & 0.04 & 1.344 & 0.047 & 1.027 \\
$\mathbf{2 1}$ & 0.079 & 1.185 & 0.073 & 1.251 \\
$\mathbf{2 2}$ & 0.044 & 1.117 & 0.041 & 1.103 \\
$\mathbf{2 3}$ & 0.073 & 1.357 & 0.074 & 1.266 \\
$\mathbf{2 4}$ & 0.025 & 1.423 & 0.028 & 1.252 \\
\hline $\mathbf{O F}$ & \multicolumn{2}{|c|}{8.0664} & \multicolumn{2}{c}{4.8} \\
& \multicolumn{2}{c}{} \\
\hline
\end{tabular}

Table 3: TMS and PS of Relays FFA and FFA-NLP 


\begin{tabular}{|c|c|c|c|c|}
\hline \multirow{2}{*}{ Relay } & \multicolumn{2}{|c|}{ Harmony Search } & \multicolumn{2}{|c|}{ HSA-NLP } \\
\cline { 2 - 5 } & TMS & PS & TMS & PS \\
\hline $\mathbf{1}$ & 0.2 & 0.684 & 0.076 & 0.738 \\
$\mathbf{2}$ & 0.153 & 0.423 & 0.045 & 0.687 \\
$\mathbf{3}$ & 0.482 & 0.052 & 0.038 & 1.499 \\
$\mathbf{4}$ & 0.128 & 0.761 & 0.038 & 1.39 \\
$\mathbf{5}$ & 0.207 & 0.34 & 0.053 & 0.568 \\
$\mathbf{6}$ & 0.139 & 0.936 & 0.061 & 1.067 \\
$\mathbf{7}$ & 0.172 & 0.744 & 0.059 & 1.165 \\
$\mathbf{8}$ & 0.122 & 0.609 & 0.044 & 0.792 \\
$\mathbf{9}$ & 0.092 & 0.847 & 0.048 & 1 \\
$\mathbf{1 0}$ & 0.14 & 0.694 & 0.054 & 0.884 \\
$\mathbf{1 1}$ & 0.138 & 0.5 & 0.054 & 0.514 \\
$\mathbf{1 2}$ & 0.118 & 0.334 & 0.058 & 0.768 \\
$\mathbf{1 3}$ & 0.412 & 0.119 & 0.07 & 0.666 \\
$\mathbf{1 4}$ & 0.395 & 0.155 & 0.053 & 1.331 \\
$\mathbf{1 5}$ & 0.422 & 0.106 & 0.071 & 0.745 \\
$\mathbf{1 6}$ & 0.314 & 0.242 & 0.07 & 0.681 \\
$\mathbf{1 7}$ & 0.067 & 1.476 & 0.073 & 1.248 \\
$\mathbf{1 8}$ & 0.109 & 1.607 & 0.032 & 1.498 \\
$\mathbf{1 9}$ & 0.072 & 1.408 & 0.075 & 1.143 \\
$\mathbf{2 0}$ & 0.159 & 1.282 & 0.037 & 1.483 \\
$\mathbf{2 1}$ & 0.077 & 1.398 & 0.074 & 1.231 \\
$\mathbf{2 2}$ & 0.146 & 1.264 & 0.028 & 1.746 \\
$\mathbf{2 3}$ & 0.059 & 1.535 & 0.073 & 1.293 \\
$\mathbf{2 4}$ & 0.107 & 1.314 & 0.027 & 1.313 \\
\hline $\mathbf{O F}$ & \multicolumn{2}{|c|}{12.2381} & \multicolumn{2}{|c}{4} \\
\hline
\end{tabular}

Table 4: TMS and PS of Relays HSA and HSA-NLP

It is somewhat more time consuming in parameter tuning of HSA as it has some additional number of parameters to be tuned simultaneously like i) HMS (harmony memory size), ii) HMCR (harmony consideration rate), iii) PARmin (minimum pitch adjusting rate), iv) PARmax (maximum pitch adjusting rate), v) BWmin (minimum bandwidth), vi) BWmax (maximum bandwidth).

\begin{tabular}{|c|c|c|c|c|c|}
\hline Relay & GA & CSA & FFA & HS & $\begin{array}{c}\text { (GA,CS, } \\
\text { FF,HS)- } \\
\text { NLP }\end{array}$ \\
\hline 1 & 0.4369 & 0.2001 & 0.332 & 0.514 & 0.2 \\
2 & 0.2 & 0.2 & 0.475 & 0.512 & 0.2 \\
3 & 0.3754 & 0.2 & 0.34 & 0.688 & 0.2 \\
4 & 0.4362 & 0.2 & 0.603 & 0.457 & 0.2 \\
5 & 0.4684 & 0.2001 & 0.406 & 0.603 & 0.2 \\
6 & 0.3464 & 0.2 & 0.545 & 0.424 & 0.2 \\
7 & 0.3058 & 0.2 & 0.413 & 0.477 & 0.2 \\
8 & 0.3161 & 0.2 & 0.471 & 0.475 & 0.2 \\
9 & 0.4927 & 0.2002 & 0.339 & 0.348 & 0.2 \\
10 & 0.4347 & 0.2 & 0.521 & 0.458 & 0.2 \\
11 & 0.3329 & 0.2 & 0.29 & 0.507 & 0.2 \\
12 & 1.6145 & 0.2 & 0.858 & 0.284 & 0.2 \\
13 & 0.4938 & 0.2 & 0.473 & 0.67 & 0.2 \\
14 & 0.4163 & 0.2 & 0.357 & 0.666 & 0.2 \\
15 & 0.2076 & 0.2 & 0.496 & 0.647 & 0.2 \\
16 & 0.2471 & 0.2001 & 0.636 & 0.62 & 0.2 \\
17 & 0.2225 & 0.2 & 0.246 & 0.197 & 0.2 \\
18 & 0.2 & 0.2 & 0.227 & 0.726 & 0.2 \\
19 & 0.2208 & 0.2 & 0.2 & 0.209 & 0.2 \\
20 & 0.2001 & 0.2001 & 0.215 & 0.78 & 0.2 \\
21 & 0.2232 & 0.2 & 0.207 & 0.22 & 0.2 \\
22 & 0.2004 & 0.2001 & 0.2 & 0.79 & 0.2 \\
23 & 0.2 & 0.2001 & 0.224 & 0.173 & 0.2 \\
24 & 0.3378 & 0.2 & 0.218 & 0.797 & 0.2 \\
\hline
\end{tabular}

Table 5: Time of operation primary relays 


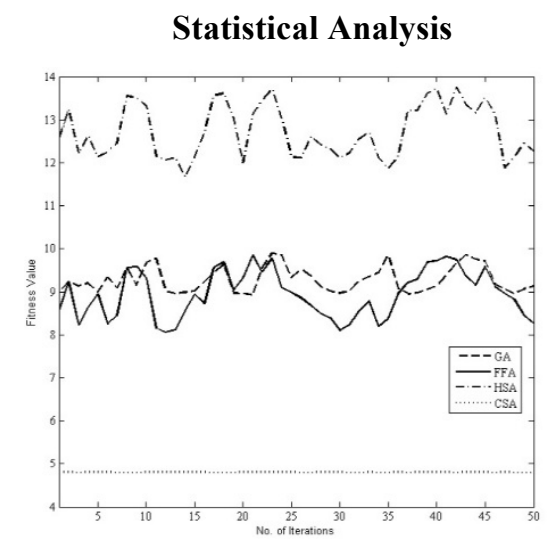

Figure 2: Statistical Analysis of 50 simulations

\begin{tabular}{|c|c|c|}
\hline Algorithms & Fitness Value & $\begin{array}{c}\text { Time of Convergence } \\
\text { (Sec.) }\end{array}$ \\
\hline Genetic Algorithm & 8.93 & 1.33 \\
\hline $\begin{array}{c}\text { Cuckoo Search } \\
\text { Algorithm }\end{array}$ & 4.8 & $420(7 \mathrm{~min}$.) \\
\hline Firefly Algorithm & 8.0664 & 20.13 \\
\hline $\begin{array}{c}\text { Harmony Search } \\
\text { Algorithm }\end{array}$ & 12.2381 & 0.8975 \\
\hline
\end{tabular}

Table 6: Comparison of Algorithms on base of fitness value and convergence time

\begin{tabular}{|c|c|c|c|c|c|}
\hline Algorithm & $\begin{array}{c}\text { Fitness } \\
\text { Value }\end{array}$ & $\begin{array}{c}\text { speed of } \\
\text { Conver- } \\
\text { gence }\end{array}$ & $\begin{array}{c}\text { Paramet } \\
\text { er } \\
\text { Tuning }\end{array}$ & $\begin{array}{c}\text { Repeat- } \\
\text { ability }\end{array}$ & Complexity \\
\hline GA & moderate & very fast & Easy & less & less \\
\hline CSA & high & very long & Easy & very high & moderate \\
\hline FFA & moderate & moderate & Tough & moderate & high \\
\hline HSA & low & Very fast & Tough & less & very high \\
\hline
\end{tabular}

Table 7: Detailed Comparison of Algorithms

\section{Conclusion}

In this paper tuned GA, CSA, FFA and HSA are productively applied for the solution of Directional overcurrent relay coordination problem. The simulations on the case study as a comparison amongst considered algorithms represented in table 6 conclude that,

Cuckoo search algorithm gives the very best and global optimum results. But the issue related with this algorithm is its very slow convergence rate. Analysis based on the time of convergence rate, the harmony search algorithm is very fast comparing to other algorithms assessed. But the drawback of this algorithm is its local best solution. The fitness value is not good compared to other algorithm. While the firefly algorithm has moderate outcome result in term of fitness value and also the time of convergence of this algorithm is also quite high approx. $20 \mathrm{sec}$. Having both the quality values of fitness value as well as less convergence time the advanced GA has the good quality over all considered algorithms.

Also from the point of view of statistical analysis CSA gives very narrow band variations while the HSA has the highest variability in outcome. 


\section{References}

1. B. Bhalja, R. P. Maheshwari and N.G. Chothani, Protection and Switchgear, 1st ed. New Delhi, India: Oxford, 2011.

2. A. Mahari and H. Seyedi, "An analytical approach for optimal coordination of overcurrent relays", IET Gen. Trans. Distr., vol. 7, no. 7, pp. 674-680, Feb. 2013.

3. H. Zeineldin, E. El-Saadany and M. Salama, "Optimal coordination of overcurrent relays using a modified particle swarm optimization,” Elect. Power Syst. Res., vol. 76, no. 11, pp. 988-995, Jan. 2006.

4. M. Mansour, S. F.Mekhamer and E.-S. N. El-Kharbawe, "A modified particle swarm optimizer for the coordination of directional overcurrent relays," IEEE Trans. Power Del., vol. 22, no. 3, pp. 1400-1410, July 2007.

5. A. S. Noghabi, J. Sadeh and H. R. Mashhadi, "Considering different network topologies inoptimal overcurrent relay coordination using a hybrid GA," IEEE Trans. Power Del., vol. 24, no. 4, pp. 1857-1863, Oct. 2009.

6. P. Bedekar and S. Bhide, "Optimum coordination of directional overcurrent relays using the hybrid GA-NLP approach," IEEE Trans. Power Del., vol. 26, no. 1, pp. 109-119, Jan. 2011.

7. M. Singh, B.K. Panigrahi, A.R. Abhyankar and S. Das, "Optimal coordination of directional over-current relays using informative differential evolution algorithm," Journal of Computational Science, vol. 5, no. 2 pp. 269-276, Mar. 2014.

8. X.-S. Yang andS. Deb, "Cuckoo Search via Lévy flights," in Proc.World Congress onNature \&Biologically Inspired Computing, 2009. NaBIC 2009., pp.210-214

9. $\quad$ D.N. Vo, P. Schegner and W. Ongsakul, "Cuckoo search algorithm for non-convex economic dispatch," IET Gen. Trans. Distr., vol.7, no.6, pp.645-654, June 2013

10. G.U. Darji, M.J. Patel, V.N. Rajput, K.S. Pandya, “A tuned cuckoo search algorithm for optimal coordination of Directional Overcurrent Relays", 2015 International Conference on Power and Advanced Control Engineering (ICPACE), Pages: 162 - 167, DOI: 10.1109/ICPACE.2015.7274936

11. Xin-She Yang; Xingshi He, "Firefly Algorithm: Recent Advances and Applications", Int. J. of Swarm Intelligence, 2013 Vol.1, No.1, pp.36 - 50

12. M. Barzegari, S. M. T Bathaee, M. Alizadeh, "Optimal coordination of directional overcurrent relays using harmony search algorithm", 9th International Conference on Environment and Electrical Engineering, pp. 321 - 324, 2010 